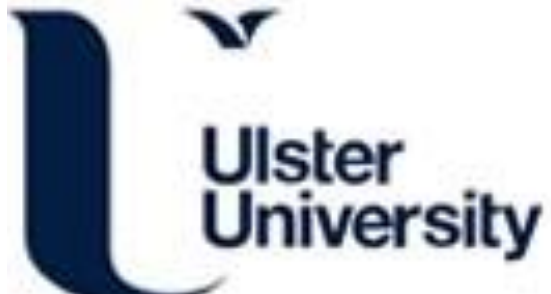

\section{Dairy intakes in older Irish adults and effects on vitamin micronutrient status: Data from the TUDA study}

Laird, E., Casey, M. C., Ward, M., Hoey, L., Hughes, C., Mccarroll, K., Cunningham, C., Strain, JJ., McNulty, H., \& Molloy, A. M. (2016). Dairy intakes in older Irish adults and effects on vitamin micronutrient status: Data from the TUDA study. The journal of nutrition, health \& aging, 21(9), 954-961. https://doi.org/10.1007/s12603-0160845-4

Link to publication record in Ulster University Research Portal

\section{Published in:}

The journal of nutrition, health \& aging

\section{Publication Status:}

Published online: 18/11/2016

DOI:

10.1007/s12603-016-0845-4

\section{Document Version}

Author Accepted version

\section{General rights}

Copyright for the publications made accessible via Ulster University's Research Portal is retained by the author(s) and / or other copyright owners and it is a condition of accessing these publications that users recognise and abide by the legal requirements associated with these rights.

\section{Take down policy}

The Research Portal is Ulster University's institutional repository that provides access to Ulster's research outputs. Every effort has been made to ensure that content in the Research Portal does not infringe any person's rights, or applicable UK laws. If you discover content in the Research Portal that you believe breaches copyright or violates any law, please contact pure-support@ulster.ac.uk. 


\title{
DAIRY INTAKES IN OLDER IRISH ADULTS AND EFFECTS ON VITAMIN MICRONUTRIENT STATUS: DATA FROM THE TUDA STUDY
}

\author{
E. LAIRD ${ }^{1}$, M.C. CASEY ${ }^{2}$, M. WARD ${ }^{3}$, L. HOEY ${ }^{3}$, C.F. HUGHES ${ }^{3}$, K. MCCARROLL ${ }^{2}$, \\ C. CUNNINGHAM ${ }^{2}$, J.J. STRAIN ${ }^{3}$, H. MCNULTY ${ }^{3}$, A.M. MOLLOY ${ }^{1,4}$
}

1. School of Biochemistry and Immunology, Trinity College Dublin, Ireland; 2. The Mercers Institute for Research on Ageing, St James's Hospital, Dublin, Ireland; 3. Northern Ireland

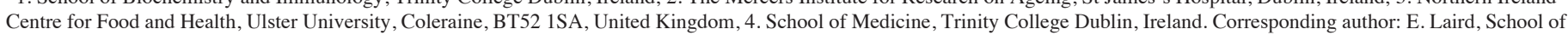
Biochemistry and Immunology, Trinity College Dublin, Ireland, lairdea@tcd.ie

\begin{abstract}
Background: Consumption of dairy products has been associated with positive health outcomes including a lower risk of hypertension, improved bone health and a reduction in the risk of type 2 diabetes. The suggested dairy intake for health in older adults is three servings per day but recent analysis of the NHANES data for older adults reported $98 \%$ were not meeting these recommendations. No studies have investigated the consequences of such declines in the dairy intakes of Irish older adults and the subsequent effects on vitamin micronutrient status. Objectives: To study the daily dairy intakes of older Irish adults and to examine how the frequency of dairy food consumption affects vitamin micronutrient status. Methods: Participants (n 4,317) were from the Trinity Ulster Department of Agriculture (TUDA) Study, a large study of older Irish adults (aged $>60 \mathrm{yrs}$ ) designed to investigate gene-nutrient interactions in the development of chronic diseases of aging. The daily intake portion for milk, cheese and yoghurt was calculated from food frequency questionnaire (FFQ) responses. Blood samples were analysed for vitamin biomarkers as follows: vitamin B12 (total serum cobalamin and holotranscobalamin (holoTC)), folate (red cell folate (RCF) and serum folate), vitamin B2 (erythrocyte glutathione reductase activation coefficient (EGRac)), vitamin B6 (serum pyridoxal phosphate) and vitamin D (serum 25(OH)D). Results: The mean total reported dairy intake was 1.16 (SD 0.79) portions per day with males consuming significantly fewer total dairy portions compared to females (1.07 vs 1.21 respectively) $(\mathrm{P}<0.05)$. There was no significant difference in total daily dairy serving intakes by age decade $(60-69,70-79$, $>80 \mathrm{yrs}$ ). Overall, only $3.5 \%$ of the total population (n 151) achieved the recommended daily dairy intake of three or more servings per day. A significantly higher proportion of females (4\%) compared to males $(2.4 \%)$ met these dairy requirements $(\mathrm{P}=0.011)$. Blood concentrations of vitamin $\mathrm{B} 12$ biomarkers, $\mathrm{RCF}$, vitamin $\mathrm{B} 2$ and vitamin B6 were significantly worse in those with the lowest tertile of dairy intake (0-0.71 servings) compared to those in the highest tertile $(1.50-4.50$ servings $)(\mathrm{P}<0.05)$. Conclusion: This study found that more than $96 \%$ of the older adults sampled did not meet current daily dairy intake recommendations. The study is the largest to-date examining dairy intakes in older Irish adults, and provides evidence that daily dairy intakes (in particular yogurt) contribute significantly to the B-vitamin and vitamin D biomarker status of older adults. These results suggest that older adults who are already vulnerable to micronutrient inadequacies, are forgoing the nutritional advantages of vitamin-rich dairy products.
\end{abstract}

Key words: Dairy intakes, yogurt, older adults, micronutrients, nutrition, vitamins.

\section{Introduction}

In Western populations, national surveys show that the proportion of older adults (defined as $>65 \mathrm{yrs}$ ) is increasing. In 2011 , older adults represented $11.4 \%(536,000)$ of the total Irish population, this has been estimated to increase to $22.4 \%$ (1.39 million) by the year 2041 (1). With such profound shifts in population demographics, also comes the corresponding rise in the frequency of age-onset chronic diseases such as osteoporosis, cardiovascular disease and diabetes (2). Evidence suggests that adequate nutritional status is one of the factors that can delay the onset of such conditions and thus the improvement of diet quality in older adults could be a cost effective health strategy (3-6). However, improving nutritional status can be problematic because of age-related conditions such as malabsorption, impaired sensory perception, increased disability and higher micronutrient requirements (7-9). Apart from food groups such as lean meats (including meat, poultry Received April 26, 2016

Accepted for publication June 29, 2016 and fish) and dairy, few of the foods that are frequently consumed by older adults provide enough of the protein and micronutrients required to maintain health (10-11).

Dairy consumption primarily comprises of milk, milk based products, yogurt and cheese and is considered an important provider of protein as well as vitamins and minerals including calcium, zinc, magnesium, vitamins $\mathrm{A}$ and $\mathrm{D}$ and the B-vitamins (11-13). However, each dairy component has varied micronutrient compositions; for example per $100 \mathrm{~g}$ on average, whole milk contains $8 \mu \mathrm{g}$ of folate, processed cheese contains $12 \mu \mathrm{g}$ while plain yogurt contains $18 \mu \mathrm{g}$ (14). Importantly, dairy products are the primary source of calcium across most industrialised countries including Europe, the USA and the UK $(11,13,15,16)$. Furthermore, consumption of dairy products has been associated with a number of positive health outcomes including a lower risk of hypertension, improved bone health and a reduction in the risk of type 2 diabetes and metabolic syndrome (17-20). Yet despite the reported health benefits, 


\section{DAIRY INTAKES IN OLDER IRISH ADULTS AND EFFECTS ON VITAMIN MICRONUTRIENT STATUS}

intakes of dairy products are not meeting the recommendations of three servings per day (21). In the USA NHANES study (2005-2006), the mean daily dairy intake of milk and yogurt for all adults was just 1.02 servings $(13,22)$. In recent findings from the National Adult Nutrition Survey (NANS) of older Irish adults ( $>65 \mathrm{yrs} ; \mathrm{n} 226)$ the mean daily dairy intake was only 1.92 servings (23). These reports are not surprising as the trend to reduce dairy intakes has increased in recent years from a myriad of factors including health concerns over certain dairy components (e.g. saturated fatty acid); the decrease in family meal consumption and more recently, to meet climate change targets $(7,9,13,24)$.

Few studies have investigated the consequences of these pressures on the dairy intakes of older adults and the subsequent effects on vitamin micronutrient status within this vulnerable sub-group. We hypothesize that low dairy intakes in this population would be associated with lower blood concentrations of micronutrients. Therefore, the aims of this study were to: firstly, detail the daily dairy intakes of milk, yogurt and cheese in older Irish adults; and second, to examine how the frequency of dairy food consumption affects the blood concentration of nutrient biomarkers.

\section{Material and methods}

\section{Study population}

Data analyzed for this study originated from the Trinity Ulster Department of Agriculture (TUDA) aging cohort study, a large study of older Irish adults ( $>60 \mathrm{yrs}$ ) designed to investigate nutritional factors, related gene-nutrient interactions and a range of health and lifestyle factors in the development of chronic diseases of aging in non-institutionalized adults. Community-dwelling participants were recruited between December 2008 and September 2012 with recruitment focused on three common diseases of aging; hypertension, cognitive dysfunction and osteoporosis. Participants were eligible for recruitment if they were aged $\geq 60$ years, without a diagnosis of dementia and their parents were ethnically Irish. Further details of sampling and recruitment have been described elsewhere $(25,26)$. Of the 5,186 participants recruited, 3 with severe frailty (Physical self-maintenance questionnaire (replied no or missing answer to the self-feeding question) and 866 with cognitive impairment (Mini-Mental State Examination (MMSE) score $<25$ ) were excluded leaving a total of 4,317 participants (Figure 1). Ethical approval was granted by the relevant authorities in each jurisdiction: the Research Ethics Committee of St. James's Hospital and The Adelaide and Meath Hospital, Dublin, and the Office for Research Ethics Committees Northern Ireland (ORECNI; reference 08/NI/RO3113) with corresponding approvals from the Northern and Western Health and Social Care Trusts, Northern Ireland. All participants provided written informed consent at the time of enrolment. All blood samples and questionnaire data were coded and identifiers removed prior to analysis.
Figure 1

Study Design

\section{Health and lifestyle questionnaire}

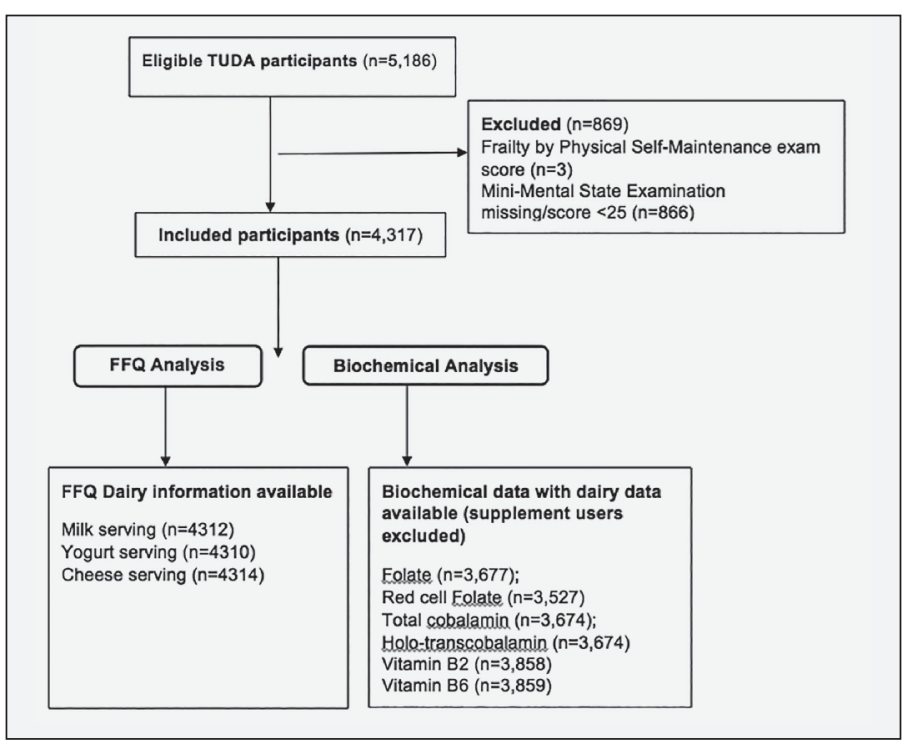

Information regarding age, sex, height, weight, medical conditions, and consumption of alcohol and smoking habits was collected. Full details of dietary supplement use (including multi-vitamins) such as dose, frequency and duration was confirmed from products or prescriptions, which participants were asked to bring along to the appointment. Where dietary supplement or drug information was unknown, the details were collected via telephone shortly after the appointment. A small number of supplements listed were unidentifiable or contained unidentifiable ingredients; individuals consuming such supplements were excluded from analysis. Those consuming vitamin supplements were not excluded in the analysis of dairy frequency intakes but were excluded from all analyses investigating the association of blood vitamin status with dairy intake servings (Figure 1).

\section{Dietary dairy intake servings}

Participants were given a modified food frequency questionnaire (FFQ) which asked if the participant consumed milk (and if so, did they consume a glass of milk), cheese and/ or yogurt and if yes how often for each dairy type (twice per day; once per day; five to six times per week; three to four times per week; one to two times per week; <once per week). The daily intake serving for milk, cheese and yogurt was calculated from the FFQ responses. The total daily dairy intake was calculated by combining the calculated daily servings of all three dairy components for each participant (Supplemental Table 1). Information was also recorded for the frequency of consumption of red meat and poultry (total meat), oily and white fish (total fish) and egg intakes. No serving size information was available for the dairy intakes, however the average serving intake size in a representative study of older 
THE JOURNAL OF NUTRITION, HEALTH \& AGINGC

Table 1

Demographic and health characteristics of the TUDA population by gender ${ }^{1-3}$

\begin{tabular}{|c|c|c|c|c|}
\hline & $\begin{array}{c}\text { Total } \\
\text { (n 4317) }\end{array}$ & $\begin{array}{l}\text { Males } \\
\text { (n 1408) }\end{array}$ & $\begin{array}{l}\text { Females } \\
\text { (n 2909) }\end{array}$ & P-value \\
\hline Age (yrs) & $72.6(38.7)$ & $72.2(7.8)$ & $72.8(8.0)$ & 0.008 \\
\hline $\mathrm{n}(\%) 60-69$ & $1691(39.2)$ & $566(40.2)$ & 1125 (38.7) & 0.336 \\
\hline $\mathrm{n}(\%) 70-79$ & $1697(39.3)$ & $566(40.2)$ & $1131(38.9)$ & 0.405 \\
\hline $\mathrm{n}(\%)>80$ & $929(21.5)$ & $276(19.6)$ & $653(22.4)$ & 0.033 \\
\hline \multicolumn{5}{|l|}{ Health \& Lifestyle } \\
\hline BMI $\left(\mathrm{kg} / \mathrm{m}^{2}\right)$ & $27.5(5.3)$ & $28.3(4.4)$ & $27.1(5.7)$ & $<0.0001$ \\
\hline GFR (ml/min) & $64.9(24.3)$ & $72.5(25.4)$ & $61.5(22.9)$ & $<0.0001$ \\
\hline Current smoker n(\%) & $515(11.9)$ & $156(11.1)$ & $359(12.3)$ & 0.228 \\
\hline Current alcohol consumer $\mathrm{n}(\%)$ & $2556(59.2)$ & $921(65.5)$ & $1635(56.2)$ & $<0.0001$ \\
\hline \multicolumn{5}{|l|}{ Supplement use $\mathrm{n}(\%)$} \\
\hline Vitamin B12 supplement user & $641(14.8)$ & $159(11.3)$ & $482(16.6)$ & $<0.0001$ \\
\hline Folic acid supplement user & $458(11.0)$ & $144(10.4)$ & $314(11.4)$ & 0.349 \\
\hline Vitamin B2 supplement user & $273(6.4)$ & $81(5.8)$ & $192(6.8)$ & 0.178 \\
\hline Vitamin B6 supplement user & $274(6.5)$ & $78(5.6)$ & $196(6.9)$ & 0.075 \\
\hline Vitamin D supplement user & $2045(47.8)$ & $449(32.0)$ & $1596(55.5)$ & $<0.0001$ \\
\hline
\end{tabular}

1. Values are means $( \pm \mathrm{SD})$ or percentage (n); 2. Independent samples T-Test for continuous variables and chi-square analysis for categorical variables for comparisons of distributions between gender; 3. GFR, golmerular filtration rate (calculated by use of the Cockcroft-Gault equation)

Irish adults (>65 yrs) (as part of the NANs Study) was 123 grams $(\mathrm{g})$ for milk, $114 \mathrm{~g}$ for yogurt and $35 \mathrm{~g}$ for cheese $(27)$.

\section{Blood collection and clinical chemistry}

A blood sample $(50 \mathrm{ml})$ was collected by venepuncture into an evacuated tube by a trained phlebotomist. Samples were kept chilled and centrifuged (3000 rpm for 15 minutes) within 3 hours of collection. After processing, samples were sent for clinical chemistry and hematological analysis. Remaining sample aliquots were stored frozen at $-80{ }^{\circ} \mathrm{C}$ until required for analysis. Kidney function tests were analysed using a Roche Cobas c701 (Roche 8000 modular system) with inter-assay CVs $<5 \%$. Glomerular filtration rate (GFR) was estimated by use of the Cockcroft-Gault equation.

\section{Nutritional biomarkers}

Serum and red cell folate (RCF) concentrations were determined by microbiological assay using a chloramphenicolresistant strain of Lactobacillus casei (28). Total cobalamin concentrations in serum were measured by a microbiological assay using a colistin-resistant strain of L.delbrueckii (29). Holotranscobalamin (holoTC) concentrations were measured by immunofluorescence using an Abbott AxSYM instrument (30). The determination of vitamin B2 (riboflavin) status was by erythrocyte glutathione reductase activation coefficient (EGRac), calculated as the ratio of flavin-dependent glutathione reductase activity before and after in vitro reactivation with its prosthetic group flavin adenine dinucleotide. Higher EGRac values were indicative of lower riboflavin status (31). Plasma pyridoxal 5-phosphate (PLP) (vitamin B6) was determined using high-performance, reverse phase, liquid chromatographic separation with detection by fluorescence (32). Vitamin D (25(OH)D (D2;D3)) concentrations were quantified using liquid chromatography-tandem mass spectrometry (API 4000; AB SCIEX; Chromsystems $\mathrm{GmbH})(25,26)$. Inter-assay CVs were as follows: serum folate and $\mathrm{RCF}<11.0 \%$, serum total cobalamin $<10.9 \%$, holo-TC $<11.1 \%$, vitamin $\mathrm{B} 2<2.2 \%$; vitamin $\mathrm{B} 6<7.1 \%$; vitamin $\mathrm{D}<5.7 \%$. The deficiency cut-offs for these biomarkers were derived from published reference ranges with $<6.8 \mathrm{nmol} / \mathrm{L}$ for serum folate; $<340 \mathrm{nmol} / \mathrm{L} \mathrm{RCF}$; $<148 \mathrm{pmol} / \mathrm{L}$ total cobalamin; $<30 \mathrm{pmol} / \mathrm{L}$ holoTC; $>1.4$ EGRac; $<20 \mathrm{nmol} / \mathrm{L} \mathrm{PLP} ;<30 \mathrm{nmol} / \mathrm{L} 25(\mathrm{OH}) \mathrm{D}(28,29,31-37)$.

\section{Statistical analysis}

Statistical analysis was performed using the Statistical Package for Social Sciences (Version 22.0; SPSS UK Ltd; Chersey, UK). Data were checked for normality and where appropriate were log-transformed. Data within the tables are expressed as means with standard deviation (SD) or as adjusted means with $95 \%$ confidence intervals. Where appropriate, an independent Student's T-test, one-way ANOVA or ANCOVA with pair-wise comparisons were applied to determine statistical differences between groups $(\mathrm{P}<0.05)$. Categorical variables were assessed by chi-square analysis while hierarchical multiple regression models were applied to determine significant predictors of nutritional biomarker concentrations. 
DAIRY INTAKES IN OLDER IRISH ADULTS AND EFFECTS ON VITAMIN MICRONUTRIENT STATUS

Table 2

Percentage of total dairy servings by sub-groups within the TUDA population ${ }^{1}$

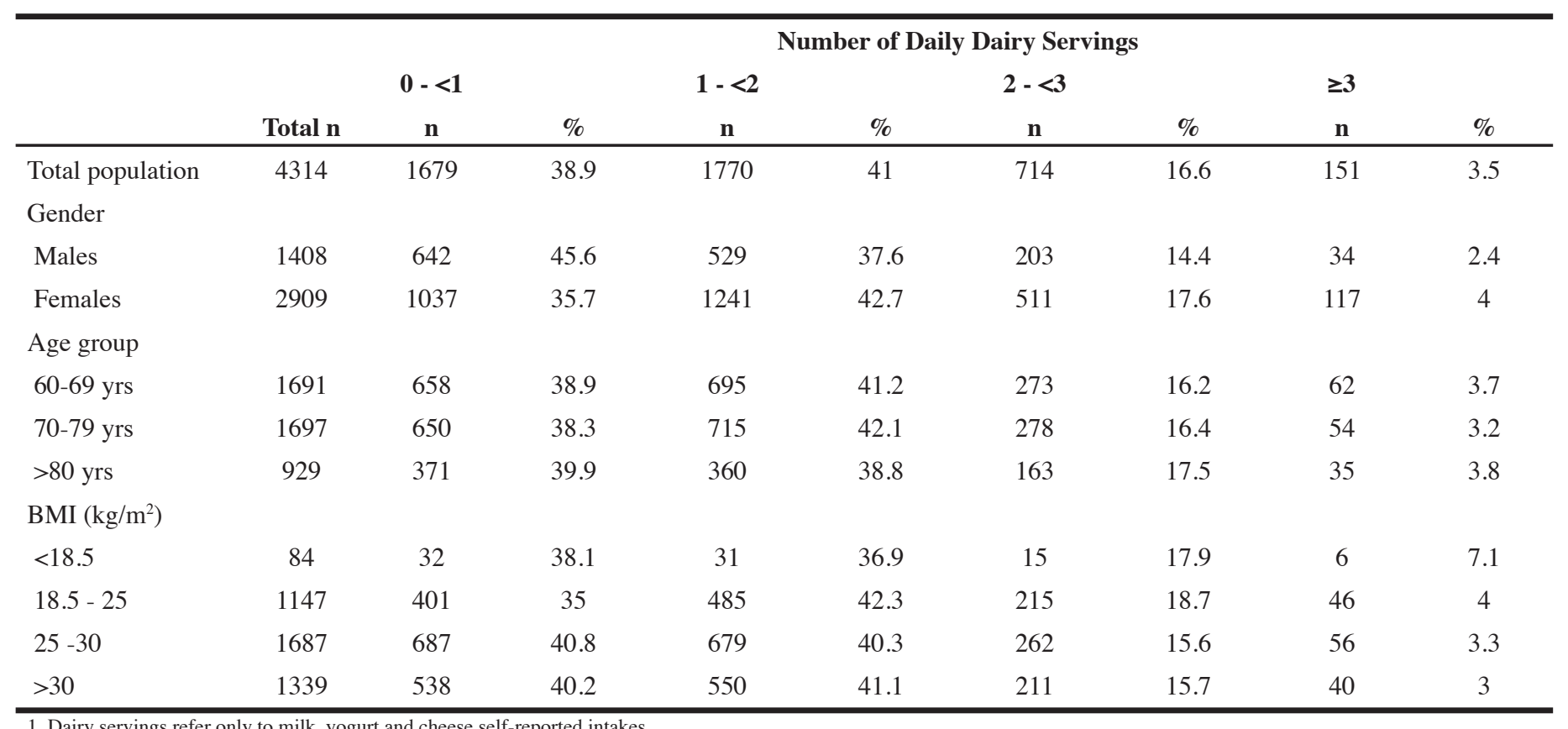

1. Dairy servings refer only to milk, yogurt and cheese self-reported intakes

\section{Results}

Baseline demographic, health and anthropometric characteristics of the TUDA population are displayed in Table 1. Total daily dairy serving data were available from 4,314 participants (1,408 males, 2,909 females). The mean age was 72.6 (SD 38.7) with a mean BMI of 27.5 (SD 5.3) kg/m2. Further detailed demographic and health characteristics of the population have been published elsewhere $(25,26)$.

\section{Daily dairy consumption}

The mean total dairy portion was 1.16 (SD 0.79) servings per day. Males reported consuming significantly less total dairy servings compared to females (1.07 vs 1.21 servings respectively) $(\mathrm{P}<0.05)$. There was no significant difference in total daily dairy servings by age. Daily servings of individual dairy components (milk, yogurt and cheese) are displayed in Supplemental Table 2. The reported mean daily milk serving significantly increased with age while the daily yogurt serving significantly decreased $(\mathrm{P}<0.05)$. The mean male daily yogurt serving was significantly lower than females ( 0.32 vs 0.42 respectively) $(\mathrm{P}<0.0001)$ while there were no significant differences in daily cheese intake servings by either age or gender.

Overall, only $3.5 \%$ of the total population ( $\mathrm{n}$ 151) achieved the recommended daily dairy intake of three or more servings per day (17) (Table 2). A significantly higher proportion of females $(4 \%)$ compared to males $(2.4 \%)$ met these dairy requirements $(\mathrm{P}=0.011)$ while a significantly higher proportion of males (45.6\%) compared to females (35.7\%) reported consuming only $0-<1$ dairy servings per day $(\mathrm{P}<0.0001)$. There were no significant differences by age-group in those achieving the recommended daily dairy serving which ranged from $3.7 \%$ for those aged 60-69 yrs to $3.8 \%$ for those aged $>80$ yrs.

\section{Nutritional biomarkers and dairy intakes}

There were significant differences in nutritional biomarker concentrations (adjusted for age, gender and BMI) across tertiles of daily dairy intake (Table 3). Biomarkers of vitamin B12 (total cobalamin; holoTC); folate (RCF); vitamin B2 (EGRac) and vitamin B6 (PLP) were all significantly poorer in those with the lowest tertile of dairy intakes (0-0.71 servings) compared to those in the highest tertile (1.50-4.50 servings) $(\mathrm{P}<0.05)$. Of the nutritional biomarkers, only the concentrations of vitamin D and serum folate did not significantly change with increasing servings. In terms of individual dairy components, participants with the highest daily intakes of milk and yogurt ( $\geq$ once per day intake serving) compared to the lowest intakes (1-2 times per week/less) had significantly higher concentrations of folate, RCF, total cobalamin, holoTC and vitamin B2 (lower EGRac concentrations) $(\mathrm{P}<0.05)$. Vitamin B6 concentrations were only significantly higher in those with the highest daily yogurt intakes $(\mathrm{P}<0.05)$. Concentrations of vitamin D did not significantly differ across frequency of milk or yogurt servings while concentrations of all nutritional biomarkers were unaffected by frequency of daily cheese servings.

In a regression model examining predictors of nutritional 
THE JOURNAL OF NUTRITION, HEALTH \& AGINGC

Table 3

Comparison of circulating nutritional biomarker concentrations across tertiles of total daily dairy intake in the TUDA population ${ }^{1,2}$

\begin{tabular}{|c|c|c|c|c|c|c|c|c|c|}
\hline & \multicolumn{9}{|c|}{ Tertile of Total Daily Dairy Servings } \\
\hline & \multicolumn{3}{|c|}{ Low $(0$ - 0.71$)$ n 1525} & \multicolumn{3}{|c|}{ Medium (0.71 - 1.50) n 1366} & \multicolumn{3}{|c|}{ High $(1.50$ - 4.50) n 1423} \\
\hline & $\mathbf{n}$ & Mean & $95 \% \mathrm{CI}$ & n & Mean & $95 \% \mathrm{CI}$ & $\mathbf{n}$ & Mean & $95 \% \mathrm{CI}$ \\
\hline \multicolumn{10}{|l|}{ Biomarker } \\
\hline Total Cobalamin (pmol/L) & 1,300 & $264^{\mathrm{a}}$ & $253-275$ & 1,180 & $282^{\mathrm{a}}$ & $271-294$ & 1,194 & $303^{\mathrm{b}}$ & $291-315$ \\
\hline HoloTC (pmol/L) & 1,300 & $61.8^{\mathrm{a}}$ & $59.1-64.6$ & 1,180 & $66.1^{\mathrm{a}}$ & $63.2-69.0$ & 1,194 & $74.6^{\mathrm{b}}$ & $71.7-77.4$ \\
\hline Serum Folate (nmol/L) & 1,303 & 31.2 & $29.4-33.1$ & 1,169 & 31.6 & $29.6-33.5$ & 1,205 & 34.4 & $32.5-36.3$ \\
\hline Red cell folate (nmol/L) & 1,250 & $974^{\mathrm{a}}$ & $946-1002$ & 1,127 & $1017^{\mathrm{a}}$ & $987-1046$ & 1,150 & $1069^{\mathrm{b}}$ & $1040-1098$ \\
\hline Riboflavin (EGRac) & 1,373 & $1.38^{\mathrm{a}}$ & $1.37-1.39$ & 1,236 & $1.34^{\mathrm{b}}$ & $1.33-1.35$ & 1,249 & $1.32^{\mathrm{c}}$ & $1.31-1.33$ \\
\hline Vitamin B6 (PLP nmol/L) & 1,372 & $69.5^{\mathrm{a}}$ & $66.8-72.2$ & 1,235 & $74.0^{\mathrm{ab}}$ & $71.1-76.8$ & 1,252 & $74.9^{\mathrm{b}}$ & $72.1-77.8$ \\
\hline Vitamin D (nmol/L) & 804 & 42.8 & $41.2-44.4$ & 644 & 44.6 & $42.8-46.4$ & 581 & 44.1 & $42.2-45.9$ \\
\hline
\end{tabular}

1. Mean values $(95 \% \mathrm{CI})$ within a row with unlike superscript letters are significantly different (using pair-wise comparisons based on estimated marginal means, adjusted for multiple comparisons (gender, age, BMI); 2. Participants receiving vitamin supplements were removed from the analysis.

Table 4

Predictors of circulating nutritional biomarker concentrations within the TUDA population ${ }^{1}$

\begin{tabular}{|c|c|c|c|c|c|c|c|c|c|c|c|c|c|c|}
\hline \multirow[b]{2}{*}{ Variable } & \multicolumn{2}{|c|}{$\begin{array}{l}\text { Total Cobala- } \\
\text { min (pmol/L) }\end{array}$} & \multicolumn{2}{|c|}{$\begin{array}{l}\text { HoloTC } \\
(\mathrm{pmol} / \mathrm{L})\end{array}$} & \multicolumn{2}{|c|}{$\begin{array}{l}\text { Serum Folate } \\
\quad(\mathrm{nmol} / \mathrm{L})\end{array}$} & \multicolumn{2}{|c|}{$\begin{array}{l}\text { Red cell Folate } \\
\quad(\mathrm{nmol} / \mathrm{L})\end{array}$} & \multicolumn{2}{|c|}{ Vitamin B2 } & \multicolumn{2}{|c|}{$\begin{array}{c}\text { Vitamin B6 } \\
(\mathrm{nmol} / \mathrm{L})\end{array}$} & \multicolumn{2}{|c|}{$\begin{array}{c}\text { Vitamin D } \\
(\mathbf{n m o l} / \mathbf{L})\end{array}$} \\
\hline & $\beta$ & P-value & $\beta$ & P-value & $\beta$ & P-value & $\beta$ & P-value & $\beta$ & P-value & $\beta$ & P-value & $\beta$ & P-value \\
\hline \multicolumn{15}{|l|}{ Daily intake } \\
\hline Milk serving & 20.64 & 0.002 & 4.92 & 0.007 & 2.01 & 0.074 & 29.97 & 0.096 & -0.02 & 0.001 & -4.45 & 0.012 & -0.26 & 0.809 \\
\hline Yogurt serving & 24.34 & 0.001 & 9.67 & $<0.001$ & 2.02 & 0.097 & 89.63 & $<0.001$ & -0.04 & $<0.001$ & 8.08 & $<0.001$ & 2.58 & 0.028 \\
\hline Cheese serving & -4.15 & 0.671 & -0.51 & 0.848 & 0.34 & 0.833 & -22.59 & 0.385 & -0.010 & 0.320 & 0.008 & 0.998 & -0.78 & 0.610 \\
\hline
\end{tabular}

biomarker concentrations, (Table 4) the daily yogurt intake was a significant predictor for concentrations of all of the nutritional biomarkers except serum folate (after adjustment for relevant co-variants). For example, each unit increase in daily yogurt intake resulted in an increase of $23.5 \mathrm{pmol} / \mathrm{L}$ of total cobalamin, $9.8 \mathrm{pmol} / \mathrm{L}$ of holoTC, $95.6 \mathrm{nmol} / \mathrm{L}$ of RCF, -0.04 $\mathrm{nmol} / \mathrm{L}$ for vitamin $\mathrm{B} 2$ (improved status), $8.9 \mathrm{nmol} / \mathrm{L}$ of vitamin B6 and $2.58 \mathrm{nmol} / \mathrm{L}$ for vitamin D. Each unit increase in daily milk servings also resulted in a significant increase in the concentrations of total cobalamin, holoTC, and an improvement in vitamin B2 status (lower EGRac concentrations). Each unit increase in daily milk servings also resulted in a decrease of $-3.9 \mathrm{nmol} / \mathrm{L}$ of vitamin B6 concentrations. The daily cheese intake was not a significant predictor for any of the nutritional biomarker concentrations.

The percentage distribution of nutrient adequacy for the circulating nutritional biomarker concentrations are shown in Supplemental Table 3. Overall, the majority of participants had either low normal or normal status for the majority of the nutritional biomarkers. The lowest distribution of nutrient deficiency was for serum folate $(2 \%$ ) which increased to $22.4 \%$ for vitamin $\mathrm{B} 2$ with the highest deficiency rate for vitamin $\mathrm{D}$ status at $31.1 \%$. The percentage of those with deficient total cobalamin status $(<148 \mathrm{pmol} / \mathrm{L})$ decreased from $16.1 \%$ in those with the lowest tertlie of total daily dairy intakes to $9 \%$ in the highest dairy intake tertile. Similar decreases in deficient vitamin biomarker status with increased total daily dairy intake were observed for vitamin B2 (26.7\% to $17.6 \%)$ and for RCF $(3.7 \%$ to $2.2 \%)$.

\section{Discussion}

This is the largest study to-date examining daily dairy intake in older adults. The findings indicate that the majority of participants sampled $(\sim 96 \%)$ did not reach the recommended guideline intake of three servings of dairy per day (21) 


\section{DAIRY INTAKES IN OLDER IRISH ADULTS AND EFFECTS ON VITAMIN MICRONUTRIENT STATUS}

regardless of age or gender. Importantly, these data demonstrate that a significant proportion of older adults who are already vulnerable to micronutrient inadequacies, are forgoing the nutritional advantages of vitamin-rich dairy products. These results also provide evidence that daily dairy servings (in particular yogurt) contribute significantly to the nutritional biomarker status of older adults.

The mean total daily dairy intake of 1.16 servings per day within this study is in line with current, albeit much smaller studies $(9,22,23,38)$. In older adults from NHANES (20072010), the mean dairy serving for those aged 51-70 yrs (n 1,771) was 1.5 per day while for those aged $>71 \mathrm{yrs}$ ( $\mathrm{n} 926$ ), it was only 1.3 servings per day (38). Furthermore, $>98 \%$ of the older adult population sampled in NHANES failed to meet the Dietary Guidelines for Americans (DGA) recommendation of three dairy servings per day (38). Similarly, data from a nationally representative sample of the Irish population (NANS), showed that $85 \%$ of older adults failed to meet the recommended dairy requirements (23). However, the NANS study reported slightly higher mean daily dairy intakes (1.92 servings) compared to NHANES or the current study. NANS was significantly smaller (n 226) than either NHANES or TUDA and was composed of a lower proportion of participants with chronic disease and frailty compared to the present study (26).

Unique to this study, females reported consuming significantly more daily dairy servings than men which was primarily driven by the difference in yogurt consumption. It was also observed that with increasing age, reported daily milk servings increased while daily yogurt servings significantly decreased. These intake trends are partly in accordance with previous dietary patterns observed in older adults however the reasons why dairy intakes change with age or gender are not clear. It has been reported that older men have a poorer awareness of healthy diet recommendations compared to women who were also more likely to have healthier dietary eating patterns $(9,39,40)$. At the same time, the older adult diet is typically characterised by a decrease in food quantity, quality and variety in comparison to younger adults $(9,41,42)$. Further research is needed to investigate these factors. Crucially, these older adult dairy intake patterns are also reflected in the nutritional blood biomarker concentrations. For instance, vitamin B6 is an important micronutrient for older adults as PLP dependant reactions are essential for amino acid biosynthesis and degradation, as well as the metabolism of neurotransmitters, such as dopamine and serotonin (43). Within this study, as age increased, yoghurt (a rich source of vitamin B6) (14) consumption decreased and was substituted for increased milk (a poorer source of vitamin B6 (14)) consumption. This could explain why increased daily milk intake and increasing age were negative predictors for PLP concentrations. At the same time, being female was also a significant positive predictor of PLP concentrations, potentially due to the gender difference in yogurt consumption.
It is important to note however, that in this study, increased milk consumption along with yogurt, was a positive predictor of a number of essential micronutrients. Both dairy components were significant positive predictors of vitamin B12 and vitamin B2 concentrations. Older adults can be at an increased risk of vitamin B12 deficiency due to gastric atrophy, medications and other factors $(44,45)$ and cobalamin deficiency can lead to the development of megaloblastic anemia and of progressive and irreversible neuropathy $(46,47)$. Inadequate vitamin B2 (riboflavin) status is also a concern for Western elderly populations (despite rich food sources available) with evidence that poor riboflavin status is associated with impaired iron metabolism and contributes to the development of anemia when iron intakes are low (48). Riboflavin has also been reported to lower blood pressure in hypertensive older adults (49). Notably, only daily yogurt intake servings alone were a positive predictors of both RCF and vitamin D concentrations. Folate is an essential component of key metabolic processes including methylation, cell proliferation and DNA replication (50) and may have benefits for cognitive aging (51), while vitamin D concentrations have been associated with a number of chronic conditions relative to aging including impaired bone health, cognition dysfunction, inflammation and cardiovascular disease $(25,52-55)$.

This is not the first study to report that dairy foods (such as milk and yoghurt) make significant contributions to the micronutrient requirements of adults. In data from day 1 of the 2003-2004 and 2005-2006 NHANES study, dairy foods contributed on average to $25 \%$ of the riboflavin, $26 \%$ of the vitamin B12 and 58\% of vitamin D dietary intakes (12). In the NANS study, dairy foods were the biggest contributor to riboflavin intakes ( $30.1 \%$ dairy; meat and fish $18.2 \%$; rice and breads 14.4\%); 2nd largest to intakes of vitamin B12 (41.6\% meat and fish; $31.7 \%$ dairy foods); 3rd largest to vitamin D intakes (meat and fish $42.9 \%$; other foods $25.9 \%$; dairy foods 9.7\%) and 4th largest to folate intakes (rice and bread $22.5 \%$; fruit and vegetables $17.2 \%$; other foods $13.9 \%$; dairy foods $13.1 \%$ ) (23). Similar to the current study, previous reports have also observed yogurt to be a particularly effective contributor to micronutrient status. In data from the Framingham Heart Study offspring cohort, yogurt consumers were 47\% and 55\% less likely to have inadequate intakes of vitamins B2 and B12 (respectively) (56) while in 2,797 Italian adults (aged 18-97 yrs), yogurt consumers were more likely to have adequate intakes of vitamins (particularly riboflavin) and minerals compared to non-consumers (57).

Unsurprisingly, reported daily cheese intake was not significantly associated with the concentrations of any nutritional biomarker as cheese is not typically fortified and does not contain sizable proportions of micronutrients (14). However, cheese servings were more frequently consumed than milk and remained unchanged between gender and age-decades. This consistency of intakes (unaffected by age or gender) could suggest that cheese is an under-utilized resource vehicle for the 


\section{THE JOURNAL OF NUTRITION, HEALTH \& AGINGC}

delivery of micronutrients through fortification. Yet this could be difficult due to older adult's health perception of cheese in terms of obesity and dietary fat $(13,58)$ despite recent studies reporting that cheese consumption does not significantly raise low density lipoprotein (LDL) cholesterol concentrations (5961). In support of this and again, comparable to the NANS population, we also observed that the highest consumers of total dairy (which included cheese) also had the lowest BMI, adding to the evidence that dairy consumption may contribute favourably to weight control, particularly in older adults (6264).

Although not measured in the current study, dairy products also contain naturally occurring bioactive peptide compounds. These peptide compounds have been reported to have antimicrobial, anti-cholesterolemic, immunomodulatory and anti-hypertensive activity within the body (65). For example, lactotripeptides such as isoleucine-proline-proline (IPP) and valine-proline-proline (VPP) have been reported to reduce systolic blood pressure (66). Results from this study suggests that older adults, who are the most likely to benefit from such reported health effects of these peptide compounds, could be forgoing these positive benefits due to inadequate dairy intakes.

One of the key strengths of this study is that it is the largest conducted to-date in older adults (n 4,317), using a well characterised population containing a range of the chronic diseases of aging typically found in the older adult population. This study also benefits from detailed health and lifestyle information as well as in-depth biochemical and nutritional biomarker analysis. Potential weaknesses of this study include that we were unable to verify the dairy intakes through food dairies (and thus did not have information on serving sizes) and had to rely on self-reported intakes although those with severe cognitive impairment or frailty were removed from the analysis to increase accuracy. For this analysis, we also did not have detailed information on socio-economic status which, as a potential marker of food quality, would have been valuable to correlate with daily dairy intakes.

To conclude, this study found that more than $96 \%$ of the older adults sampled did not meet current daily dairy intake recommendations. Dairy products (especially yogurt) are meaningful sources of micronutrients and contribute significantly to the B-vitamin and vitamin D biomarker status of older adults. These results emphasize the need for increasing awareness and education among older adults (and in particular males), on the importance of dairy intakes for health. They also highlight opportunities for the food industry in developing micronutrient rich, acceptable dairy products for the elderly consumer. The potential benefits of dairy for weight control in older adults also warrants further investigation.

Ethics declaration: All experimental procedures were conducted in accordance with the guidelines in the Declaration of Helsinki and approved by the Ethics Committee of University of Ulster and St James's Hospital Dublin.

Acknowledgement of Funding: Funding to conduct this work was provided by the Irish Department of Agriculture, Food and the Marine through the grants 07FHRIUCD1
(«JINGO» 2007-2013) and 13F407 («JINGO-JPI»/»ENPADASI» 2014-2016). Funding was also provided from The National Dairy Council (NDC) Ireland.

Disclosure: Dr. Laird reports grants from Irish Department of Agriculture, Food and the Marine, grants from The National Dairy Council (NDC) Ireland, during the conduct of the study. Dr. Casey has nothing to disclose. Dr. Ward has nothing to disclose. Dr. Hoey has nothing to disclose. Dr. Hughes has nothing to disclose. Dr. McCarroll has nothing to disclose. Dr. Cunningham has nothing to disclose. Dr. Strain has nothing to disclose. Dr. McNulty has nothing to disclose. Dr. Molloy has nothing to disclose.

\section{References}

1. Central Statistics Office (2013). Population and labour force projections. Stationery Office, Dublin. http://www.cso.ie/en/media/csoie/releasespublications/documents. Accessed 8th March 2016.

2. Lim SS, Vos T, Flaxman AD, et al. A comparative risk assessment of burden of disease and injury attributable to 67 risk factors and risk factor clusters in 21 regions, 1990-2010: a systematic analysis for the Global Burden of Disease Study 2010. Lancet 2012;380: 2224-2260.

3. Reedy J, Krebs-Smith SM, Miller PE, et al. Higher diet quality is associated with decreased risk of all-cause, cardiovascular disease, and cancer mortality among older adults. J Nutr 2014;144: 881-889.

4. Crichton GE, Elias MF, Davey A, et al. Higher cognitive performance is prospectively associated with healthy dietary choices: the Maine Syracuse Longitudinal Study. J Prev Alzheimers Dis 2015;2: 24-32.

5. Rabassa M, Zamora-Ros R, Andres-Lacueva C, et al. Association between both total baseline urinary and dietary polyphenols and substantial physical performance decline risk in older adults: A 9-year follow-up of the InCHIANTI study. J Nutr Health Aging 2016;20: 478-484.

6. Nijholt W, Jager-Wittenaar H, Visser M, et al. Are a healthy diet and physical activity synergistically associated with cognitive functioning in older adults? J Nutr Health Aging 2016;20: 525-532.

7. Chen $\mathrm{CCH}$, Schilling $\mathrm{IS}$, lyder $\mathrm{CH}$. A concept analysis of malnutrition in the elderly. J adv nurs 2008;36: 131-142.

8. Brownie S. Why are elderly individuals at risk of nutritional deficiency? Int J nurs Pract 2006;12: 110-118.

9. Power SE, Jeffery IB, Ross RP et al. Food and Nutrient intake of Irish communitydwelling elderly subjects: Who is at nutritional risk? J Nutr Health Aging 2014;18: 561-572.

10. McNeill S, Van Elswyk ME. Red meat in global nutrition. Meat Science 2012;92: 166-173.

11. Prentice AM. Dairy products in global public health. Am J Clin Nutr 2014;99: 1212S-1216S.

12. Rice BH, Quann EE, Miller GD. Meeting and exceeding dairy recommendations: effects of dairy consumption on nutrient intakes and risk of chronic disease. Nutr Rev 2013;71: 209-223.

13. Heaney, RP. Dairy intake, dietary adequacy and lactose intolerance. Adv Nutr 2013;4: 151-156.

14. Food Standards Agency. Mccance and Widdowson's the Composition of Foods Sixth Summary Edition, 6th revised ed.; Royal Society of Chemistry: Cambridge, UK, 2002.

15. Fulgoni VL, Keast DR, Auestad N, et al. Nutrients from dairy foods are difficult to replace in diets of Americans: food pattern modeling and an analyses of the National Health and Nutrition Examination Survey 2003-2006. Nutr Res 2011;31: 759-765.

16. Rafferty K \& Heaney RP. Nutrient effects on the calcium economy: Emphasizing the potassium controversy. J Nutr 2008;138: 166S-171S

17. Tong X, Dong JY, Wu ZW, et al. Dairy consumption and risk of type 2 diabetes mellitus: a meta-analysis of cohort studies. Eur J Clin Nutr 2011;65: 1027-1031.

18. Rizzoli R. Dairy products, yogurts and bone health. Am J Clin Nutr 2014;99: 1256S-1262S.

19. Elwood PC, Givens DI, Beswick AD, et al. The survival advantage of milk and dairy consumption: an overview from cohort studies of vascular diseases, diabetes and cancer. J Am Coll Nutr 2008;27: 723S-734S.

20. Ralston RA, Lee JH, Truby H, et al. A systematic review and meta-analysis of elevated blood pressure and consumption of dairy foods. J Hum Hypertens 2012;26: 3-13.

21. Department of Health, Ireland (2012). Your Guide to Healthy Eating Using the Food Pyramid, Health Service Executive.

22. Dairy Research Institute (NHANES 2005-2008). Data Source: Centers for Disease Control and Prevention, National Center for Health Statistics, National Health and Nutrition Examination Survey Data. Hyattsville, MD: U.S. Department of Health and Human Services, Centers for Disease Control and Prevention, [2005-2006, 2007-2008]. http://www.cdc.gov/nchs/nhanes.htm. Accessed 9th March 2016

23. Irish Universities Nutrition Alliance (IUNA) (2014). Report on the Contribution of Dairy Foods to the Nutritional Quality of the Diet in Older Irish Adults (Aged 65 years and older) 


\section{DAIRY INTAKES IN OLDER IRISH ADULTS AND EFFECTS ON VITAMIN MICRONUTRIENT STATUS}

24. Bryngelsson D, Wirsenius S, Hedenus F et al. How can the EU climate targets be met? A Combined analysis of technological and demand-side changes in food and agriculture. Food Policy 2016;59: 152-164.

25. Laird E, McNulty H, Ward M, et al. Vitamin D deficiency is associated with inflammation in older Irish adults. J Clin Endocrinol Metab. 2014;99: 1807-1815.

26. McCarroll K, Beirne A, Casey M, et al. Determinants of 25-hydroxyvitamin D in older Irish adults. Age Ageing. 2015;44: 847-853.

27. Irish Universities Nutrition Alliance (IUNA) (2013). The Irish food portion sizes database (1st edition).

28. Molloy AM, Scott JM. Microbiological assay for serum, plasma, and red cell folate using cryopreserved, microtiter plate method. Methods Enzymol. 1997;281: 43-53.

29. Kelleher B, Broin S. Microbiological assay for vitamin B12 performed in 96-well microtitre plates. Journal of clinical pathology. 1991;7: 592-595.

30. Brady J, Wilson L, McGregor L, et al. Active B12: a rapid, automated assay for holotranscobalamin on the Abbott AxSYM analyzer. Clin Chem. 2008;54: 567-573.

31. Powers HJ, Bates CJ, Prentice AM, et al. The relative effectiveness of iron and iron with riboflavin in correcting a microcytic anaemia in men and children in rura gambia. Hum Nutr Clin Nutr 1983;37: 413-425.

32. Bates CJ, Pentieva KD, Matthews N, et al. A simple, sensitive and reproducible assay for pyridoxal 5'-phosphate and 4-pyridoxic acid in human plasma. Clin Chim Acta 1999;280: 101-111.

33. Pfeiffer CM, Caudill SP, Gunter EW, et al. Biochemical indicators of B vitamin status in the US population after folic acid fortification: results from the Nationa Health and Nutrition Examination Survey 1999-2000. Am J Clin Nutr. 2005;2: 442 450

34. Selhub J, Morris MS, Jacques PF. In vitamin B12 deficiency, higher serum folate is associated with increased total homocysteine and methylmalonic acid concentrations. Proc Natl Acad Sci USA. 2007;50:19995-20000.

35. Bailey RL, Carmel R, Green R, et al. Monitoring of vitamin B-12 nutritional status in the United States by using plasma methylmalonic acid and serum vitamin B-12. Am J Clin Nutr. 2011;2: 552-561.

36. Valente E, Scott JM, Ueland PM et al. Diagnostic accuracy of holotranscobalamin, methylmalonic acid, serum cobalamin, and other indicators of tissue vitamin B12 status in the elderly. Clin Chem. 2011;6: 856-63.

37. Institute of Medicine Food and Nutrition Board. Dietary Reference Intakes for Calcium and Vitamin D. Washington, DC: National Academy Press, 2011.

38. Quann EE, Fulgoni VL (III), Auestad N. Consuming the daily recommended amounts of dairy products would reduce the prevalence of inadequate micronutrient intakes in the United States: diet modeling study based on NHANES 2007-2010. Nutr J. 2015;14: 90 .

39. Bamia C, Orfanos P, Ferrari P et al. dietary patterns among older Europeans: the EPIC-Elderly study. Br J Nutr 2005;94: 100-113.

40. Baker AH \& Wardle J. Sex differences in fruit and vegetable intake in older adults. appetite 2003;40: 269-275.

41. Zhu K, Devine A, Suleska A et al. adequacy and change in nutrient and food intakes with aging in a seven-year cohort study in elderly women. J Nutr Health Aging 2010;14: 723-729.

42. Kwon J, Suzuki T, Kumagai S et al. Risk factors for dietary variety decline among Japanese elderly in a rural community: A 8-year follow-up study from TMIG-LISA. Eur J Clin Nutr 2005;60: 305-311.

43. Ueland, PM, Ulvik A, Rios-Avila L et al. Direct and functional biomarkers of vitamin B6 status. Annu Rev Nutr. 2015;35: 33-70.

44. Dharmarajan T, Adiga G, Norkus EP. Vitamin B12 deficiency. Recognizing subtle symptoms in older adults. Geriatrics. 2003;3: 30-4, 7-8.

45. Lam JR, Schneider JL, Zhao W, Corley DA. Proton pump inhibitor and histamine 2 receptor antagonist use and vitamin B12 deficiency. Jama. 2013;22: 2435-2442.
46. Stabler SP. Vitamin B12 deficiency. N Engl J of Med. 2013;368(2):149-60.

47. Savage DG, Lindenbaum J. Neurological complications of acquired cobalamin deficiency: clinical aspects. Baillieres Clin Haematol. 1995;3: 657-678.

48. Powers HJ. Riboflavin (vitamin B2) and health. Am J Clin Nutr. 2003;77: 1352-1360.

49. Wilson $\mathrm{CP}$, McNulty $\mathrm{H}$, Ward M, et al. Blood pressure in treated hypertensive individuals with the MTHFR 677TT genotype is responsive to intervention with riboflavin: findings of a targeted randomized trial. Hypertension 2013, 61: 13021308.

50. Bailey LB, Gregory JF. Folate metabolism and requirements. The Journal of nutrition. 1999;4: 779-782.

51. Horvat P, Gardiner J, Kubinova $\mathrm{R}$ et al. Serum folate, vitamin B12 and cognitive function in middle and older age: The HAPIEE study. Exp Gerontol. 2016;76: 33-38.

52. Tang B, Eslick GD, Nowson C, et al. Use of calcium or calcium in combination with vitamin D supplementation to prevent fractures and bone loss in older people: a metaanalysis. Lancet. 2007;370: 657-666.

53. Balion C, Griffith LE, Strifler L, et al. Vitamin D, cognition, and dementia: a systematic review and meta-analysis. Neurology 2012;79: 1397-1405.

54. Pludowski P, Holick MF, Pilz S, et al. Vitamin D effects on musculoskeletal health, immunity, autoimmunity, cardiovascular disease, cancer, fertility, pregnancy, dementia and mortality: A review of recent evidence. Autoimmun Rev. 2013;10: 976989.

55. Wang TJ, Pencina MJ, Booth SL, et al. Vitamin D deficiency and risk of cardiovascular disease. Circulation. 2008;117: 503-511.

56. Wang H, Livingston KA, Fox CS, et al. Yogurt consumption is associated with better diet quality and metabolic profile in American men and women. Nutr Res. 2013; 33 $18-26$

57. Mistura L, D'Addezio L, Sette S, et al. Diet quality of Italian yogurt consumers: an application of the probability of adequate nutrient intake score (PANDiet). Int J Food Sci Nut. 2016;24: 1-7.

58. Chollet M, Gille D, Piccinali P, et al. Dairy consumption among middle aged and elderly adults in Switzerland. J Dairy Sci. 2014; 97: 5387-5392.

59. Biong AS PC et al. A comparison of the effects of cheese and butter on serum lipids, haemostatic variables and homocysteine. Br J Nutr. 2004; 92: 791-797.

60. Hjerpsted J, Leedo E, Tholstrup T. Cheese intake in large amounts lowers LDLcholesterol concentrations compared with butter intake of equal fat content. Am J Clin Nutr. 2011; 94: 1479-1484.

61. Nilsen R, Høstmark AT, Haug A, et al. Effect of a high intake of cheese oncholesterol and metabolic syndrome: results of a randomized trial. Food Nutr Res. 2015;59.

62. Abargouei AS, Janghorbani M, Salehi-Marzijarani M, et al. Effect of dairy consumption on weight and body composition in adults: a systematic review and meta-analysis of randomized controlled clinical trials. Int J Obes (Lond). 2012 36: 1485-1493.

63. Wang H, Troy LM, Rogers GT, et al. Longitudinal association between dairy consumption and changes of body weight and waist circumference: the Framingham Heart Study. Int J Obes (Lond). 2014 38: 299-305.

64. Dairy consumption in association with weight change and risk of becoming overweight or obese in middle-aged and older women: a prospective cohort study. Am J Clin Nutr. 2016;4: 979-988.

65. Mohanty DP, Mohapatra S, Misra S, et al. Milk derived bioactive peptides and their impact on human health-A review. Saudi Journal of Biological Sciences (2015).

66. Cicero AFG et al. Do the lactotripeptides isoleucine-proline-proline and valineproline-proline reduce systolic blood pressure in European subjects? A meta-analysis of randomized controlled trials. Am J Hypertens. 2013; 26: 442-449. 